\title{
Magnetic field measurements at milliarcsecond resolution around massive young stellar objects.
}

\author{
Gabriele SURCIS* \\ Joint Institute for VLBI in Europe \\ E-mail: surcisejive.nl
}

\author{
Wouter H.T. VLEMMINGS \\ Chalmers University of Technology, Onsala Space Observatory \\ E-mail: wouter.vlemmings@chalmers.se
}

\section{Huib J. VAN LANGEVELDE}

Joint Institute for VLBI in Europe and Sterrewacht Leiden, Leiden University

E-mail: langevelde@jive.nl

\section{Busaba HUTAWARAKORN KRAMER}

Max-Planck Institut für Radioastronomie

E-mail: bkramer@mpifr-bonn.mpg • de

\section{Anna BARTKIEWICZ}

Centre for Astronomy, Faculty of Physics, Astronomy and Informatics, Nicolaus Copernicus University

E-mail: annaneastro.uni.torun.pl

\section{Hans ENGELKAMP}

High Field Magnet Laboratory, Institute for Molecules and Materials, Radboud University Nijmegen

E-mail: h.engelkampescience.ru.nl

Magnetic fields have only recently been included in theoretical simulations of high-mass star formation. The simulations show that magnetic fields can play a crucial role not only in the formation and dynamics of molecular outflows, but also in the evolution of circumstellar disks. Therefore, new measurements of magnetic fields at milliarcsecond resolution close to massive young stellar objects (YSOs) are fundamental for providing new input for numerical simulations and for understanding the formation process of massive stars. The polarized emission of 6.7 $\mathrm{GHz} \mathrm{CH}_{3} \mathrm{OH}$ masers allows us to investigate the magnetic field close to the massive YSO where the outflows and disks are formed. Recently, we have detected with the EVN $\mathrm{CH}_{3} \mathrm{OH}$ maser polarized emission towards 10 massive YSOs. From a first statistical analysis we have found evidence that magnetic fields are primarily oriented along the molecular outflows. To improve our statistics we are carrying on a large observational EVN campaign for a total of 19 sources, the preliminary results of the first seven sources are presented in this contribution. Furthermore, we also describe our efforts to estimate the Landé $g$-factors of the $\mathrm{CH}_{3} \mathrm{OH}$ maser transition to determine the magnetic field strength from our Zeeman-splitting measurements.

12th European VLBI Network Symposium and Users Meeting

7-10 October 2014

Cagliari, Italy

\footnotetext{
* Speaker.
} 


\section{Introduction}

The whole process that governs low-mass star formation $\left(\mathrm{M}<8 \mathrm{M}_{\odot}\right)$ is nowadays thought to be fairly well understood. The low-mass stars are formed through gravitational collapse of bound cores that are created by the fragmentation of molecular clouds. During the formation of the star an accretion disk around the central protostar is formed and a jet/bipolar outflow is launched perpendicular to the disk. Furthermore, the magnetic field is thought to play an important role in slowing the collapse, in transferring the angular momentum, and in powering the outflow (e.g., [1]). However, there still exists an open debate on whether the orientation of the magnetic field aligns with respect to the orientation of molecular outflow. Recently, two independent surveys of dust polarized emission towards low-mass protostellar cores showed opposing results. One found no correlation between magnetic field orientation and outflow axis in low-mass young stellar objects (YSOs) [2], while the other one found a good alignment [3].

The core accretion model describes the formation of high-mass stars $\left(M>8 \mathrm{M}_{\odot}\right)$ as a scaledup version of the low-mass star formation (e.g., [4]). Recent theoretical simulations have suggested that magnetic fields might play a role in massive star formation as important as in low-mass star formation. Indeed, the simulations begin to reproduce the observations only when the magnetic field is taken into consideration (e.g., [5], [6], [7]). Similarly to the low-mass star formation case, conflicting results on the orientation of the magnetic field with respect to the outflow orientation have also been found. Based on the observations of dust polarized emission towards a sample of 21 sources, no correlation between outflow axis and magnetic field orientation has been found at arcsecond resolution $\left(\sim 10^{3} \mathrm{au}\right)$ in massive star-forming regions (SFRs) [8]. On the contrary, by observing the polarized emission of $6.7 \mathrm{GHz} \mathrm{CH} \mathrm{CH}_{3} \mathrm{OH}$ masers with the European VLBI Network ${ }^{1}$ we have found evidence that on scales of 10-100 au magnetic field around massive YSOs is preferentially oriented along the outflow [9]. This is supported by a Kolmogorov-Smirnov (K-S) test performed on a sample of nine sources that shows a probability of $10 \%$ that the distribution of the projected angles $\left|\mathrm{PA}_{\text {outflow }}-\left\langle\Phi_{\mathrm{B}}\right\rangle\right|$ is drawn from a random distribution (see Fig. 1). Here, $\mathrm{PA}_{\text {outflow }}$ is the orientation of the outflow and $\left\langle\Phi_{\mathrm{B}}\right\rangle$ is the error weighted orientation of the magnetic field [9].

Furthermore, providing new measurements of magnetic fields strength at mas resolution close to the massive YSOs by measuring the Zeeman splitting of the $6.7 \mathrm{GHz} \mathrm{CH}_{3} \mathrm{OH}$ maser emission is fundamental to verify and/or improve the numerical simulations of massive star formation. Even though in the last years Zeeman-splitting measurements of $\mathrm{CH}_{3} \mathrm{OH}$ maser emission have been made, the exact proportionality between the measured splitting and the magnetic field is still uncertain ([10], [11], [9]). Therefore, it is of great importance to measure the still unknown Landé g-factors for the $\mathrm{CH}_{3} \mathrm{OH}$ molecule.

\section{The flux-limited sample}

To improve our statistics it is important to enlarge the number of massive SFRs toward which $\left|\mathrm{PA}_{\text {outflow }}-\left\langle\Phi_{\mathrm{B}}\right\rangle\right|$ can be measured; in other words, we need to enlarge the number of massive

\footnotetext{
${ }^{1}$ The European VLBI Network is a joint facility of European, Chinese, South African and other radio astronomy institutes funded by their national research councils.
} 

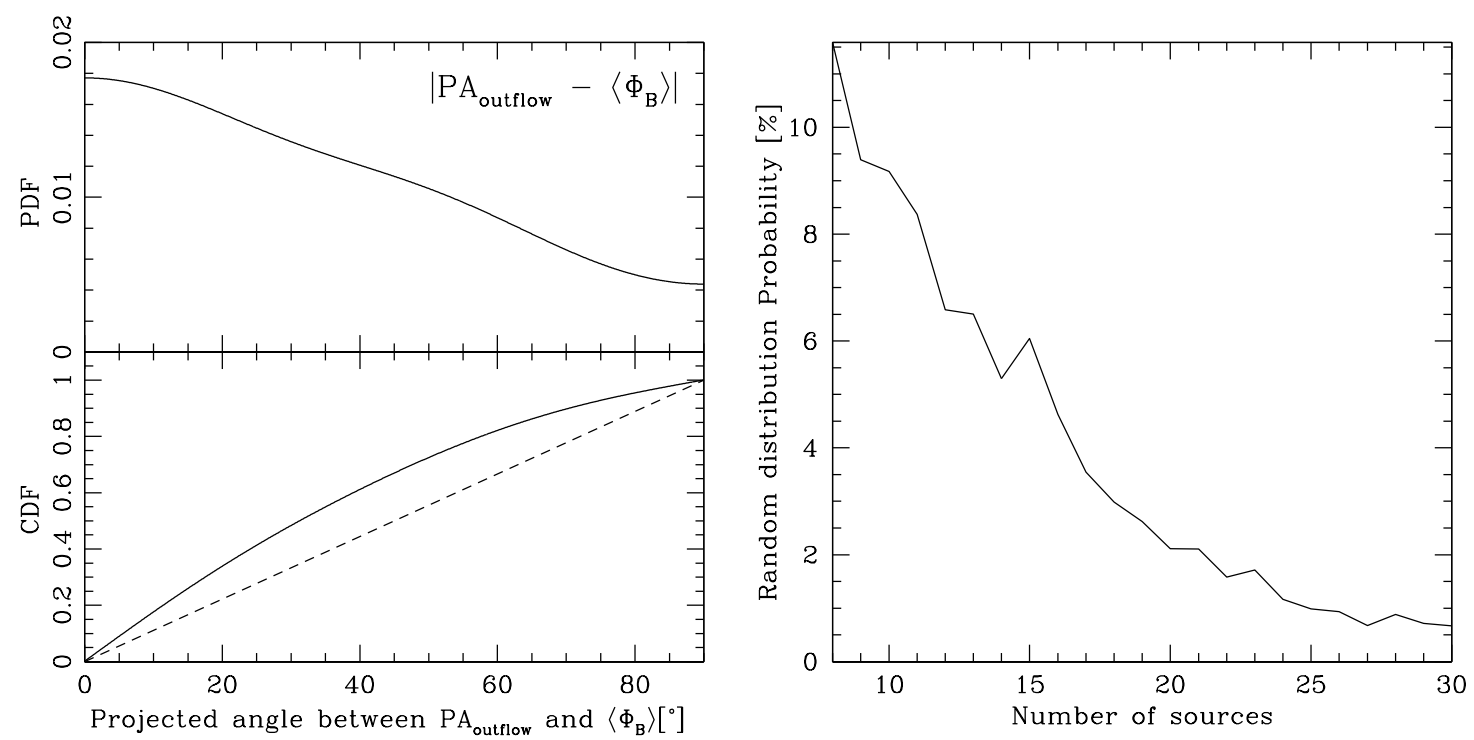

Figure 1: Left. The probability distribution function (PDF, top panel) and the cumulative distribution function (CDF, bottom panel) of the projected angle between the outflow axes and the magnetic field $\left(\left|\mathrm{PA}_{\text {outflow }}-\left\langle\Phi_{\mathrm{B}}\right\rangle\right|\right)$ based on nine sources as measured by [9]. The dashed line is the CDF for random orientation of outflows and magnetic fields, i.e. all angular differences are equally likely. Right. The random distribution probability of $\left|\mathrm{PA}_{\text {outflow }}-\left\langle\Phi_{\mathrm{B}}\right\rangle\right|$ determined with a K-S test as function of number of sources.

YSOs for which the orientation of the magnetic field at milliarcsecond (mas) resolution is measured. With a Monte-Carlo simulation we determined the number of sources that we would need to significantly decrease the K-S probability, taking into account observational errors in the outflow and magnetic field angles. Hence we determined the K-S probability between 8 and 30 vectors pairs (or sources). The vectors in a pair represent $\mathrm{PA}_{\text {outflow }}$ and $\left\langle\Phi_{\mathrm{B}}\right\rangle$ and are randomly selected, with the only apriori condition that their $3 \mathrm{D}$ misalignment is $<30^{\circ}$. We found that the probability decreases below $1 \%$ for more than 28 sources (Fig 1).

We have selected a flux-limited sample of massive SFRs with declination $>-9^{\circ}$ and a total $\mathrm{CH}_{3} \mathrm{OH}$ maser single-dish flux greater than $50 \mathrm{Jy}$ from the $6.7 \mathrm{GHz} \mathrm{CH} \mathrm{CH}_{3} \mathrm{OH}$ maser catalogue of [12]. To detect circularly polarized $\mathrm{CH}_{3} \mathrm{OH}$ maser emission $(\leq 1 \%)$, we have excluded the regions hosting $\mathrm{CH}_{3} \mathrm{OH}$ maser that in recent single-dish observations showed a total flux below $20 \mathrm{Jy}$ [10]. The total number of massive SFRs of the flux-limited sample is thus 31 . The polarimetric $6.7 \mathrm{GHz}$ $\mathrm{CH}_{3} \mathrm{OH}$ maser observations, and the consequent magnetic field measurements, of twelve of these SFRs had already been published in recent past ([13], [14], [15], [11], [9], [16]). Therefore, 19 massive SFRs remain to be observed. We were given EVN time to observe all of them at $6.7 \mathrm{GHz}$.

To date 16 out of the 19 sources have been observed in full polarization mode at $6.7 \mathrm{GHz}$ by using eight of the EVN antennas (Effelsberg, Jodrell, Onsala, Medicina, Noto, Torun, Westerbork, and Yebes- $40 \mathrm{~m}$ ), for a total observation time of $112 \mathrm{~h}$. The remaining three sources are scheduled to be observed during the second EVN session (May-June) of 2015. The results of the first seven observed sources are briefly summarized in Sect. 3 and extensively reported in [17].

\section{Results}

We have detected a total of $176 \mathrm{CH}_{3} \mathrm{OH}$ maser features towards the first seven sources of the 
flux-limited sample (i.e., G24.78+0.08, G25.65+1.05, G29.86-0.04, G35.03+0.35, G37.43+1.51, G174.20-0.08, and G213.70-12.6). We were able to determine the orientation of the magnetic field around all the sources but G174.20-0.08, toward which no linearly polarized $6.7 \mathrm{GHz} \mathrm{CH}_{3} \mathrm{OH}$ maser emission was detected at $\geq 5 \sigma$ (i.e., $\geq 20 \mathrm{mJy}$ beam $^{-1}$ ). The magnetic field is along the outflow (with a misalignment $<30^{\circ}$ ) in three massive YSOs (G25.65+1.05, G35.03+0.35, and G213.7012.6) and is perpendicular to the outflow $\left(>75^{\circ}\right)$ in the remaining massive YSOs (G24.78+0.08, G29.86-0.04, and G37.43+1.51).

We performed a new statistical analysis by adding to the nine sources previously studied [9] the new magnetic field measurements made around the sources reported above and around IRAS 20126+4104 [16]. Moreover, in our analysis we also add two of the southern sources observed by [18] that were recently associated with CO-outflows [8]. Although the number of sources for which we measure $\left|\mathrm{PA}_{\text {outflow }}-\left\langle\Phi_{\mathrm{B}}\right\rangle\right|$ is twice than that in [9] (18 vs. 9), the K-S test still shows a probability of $10 \%$, which is three times larger than expected (3\% from Fig. 1). If the magnetic field aligns with the outflow axis, this probability difference can be due, for instance, to the selection criteria chosen to observe the first seven sources, which might not be representative of the whole sample. That is, we have by chance observed all the sources that do not show on the plane of the sky an alignment of the magnetic field w.r.t. the outflow axis. Nevertheless, because this probability is low our previous conclusion can be considered still valid, i.e. the magnetic field close to the central YSO (10-100 au) is preferentially oriented along the outflow axis. Of course, given the $10 \%$ probability, the misalignment scenario cannot be ruled out. However, before drawing any conclusion, we have to reduce and analyse the data of the 12 remaining massive SFRs.

\section{Zeeman-splitting coefficient of $\mathrm{CH}_{3} \mathrm{OH}$ maser ${ }^{2}$}

Besides determining the magnetic field orientation around massive YSOs by analyzing the linearly polarized emission of $6.7 \mathrm{GHz} \mathrm{CH} \mathrm{CH}_{3} \mathrm{OH}$ maser, we are also able to measure the Zeeman splitting of the maser line by detecting the circularly polarized emission. Considering all the sources observed with the EVN, we measured Zeeman splittings in the range $0.4 \mathrm{~ms}^{-1}<\Delta \mathrm{V}_{\mathrm{Z}}<10 \mathrm{~ms}^{-1}$ ([14], [15], [11], [9], [16]). The magnetic field strength is simply related to $\Delta V_{\mathrm{Z}}$ by $B=\frac{B_{\|}}{\cos \theta}=\frac{\Delta V_{Z}}{\alpha_{\mathrm{Z}}}$, where $\theta$ is the angle between the magnetic field and the maser propagation direction and $\alpha_{Z}$ is the Zeeman-splitting coefficient that depends on the Landé $g$-factors $\left(g_{\mathrm{L}}\right)$ of the maser emission (e.g., [10]). By modeling the linearly polarized emission we are able to estimate $\theta$ (e.g., [15]), but $\alpha_{\mathrm{Z}}$ still

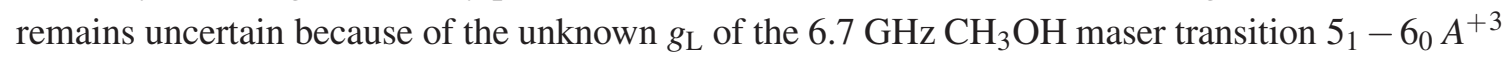
[10]. Therefore, to provide magnetic field strength values around massive YSOs it is crucial to estimate the $g_{\mathrm{L}}$ of the $\mathrm{CH}_{3} \mathrm{OH}$ transition $5_{1}-6_{0} A^{+}$by measuring in a laboratory the $g_{\mathrm{L}}$ factors of more accessible $\mathrm{CH}_{3} \mathrm{OH}$ molecule transitions or by theoretical calculations. Preferably both.

In November 2013 we set up an experiment by using one of the most powerful magnet facility in Europe, which at that time was able to reach a magnetic field of $33.4 \mathrm{~T}\left(3.34 \times 10^{5} \mathrm{G}\right)$, at the High Field Magnet Laboratory of the Radboud University (Nijmegen, the Netherlands). We tried to measure the $g_{\mathrm{L}}$ of many rotational $\mathrm{CH}_{3} \mathrm{OH} A^{+}$absorption lines in the infrared region $(0.6 \mathrm{THz}-$

\footnotetext{
${ }^{2}$ We acknowledge the support of the HFML-RU/FOM, member of the European Magnetic Field Laboratory (EMFL).

${ }^{3}$ The $\mathrm{CH}_{3} \mathrm{OH}$ molecule has two different symmetries $\mathrm{E} 1$ and $A^{+}$.
} 


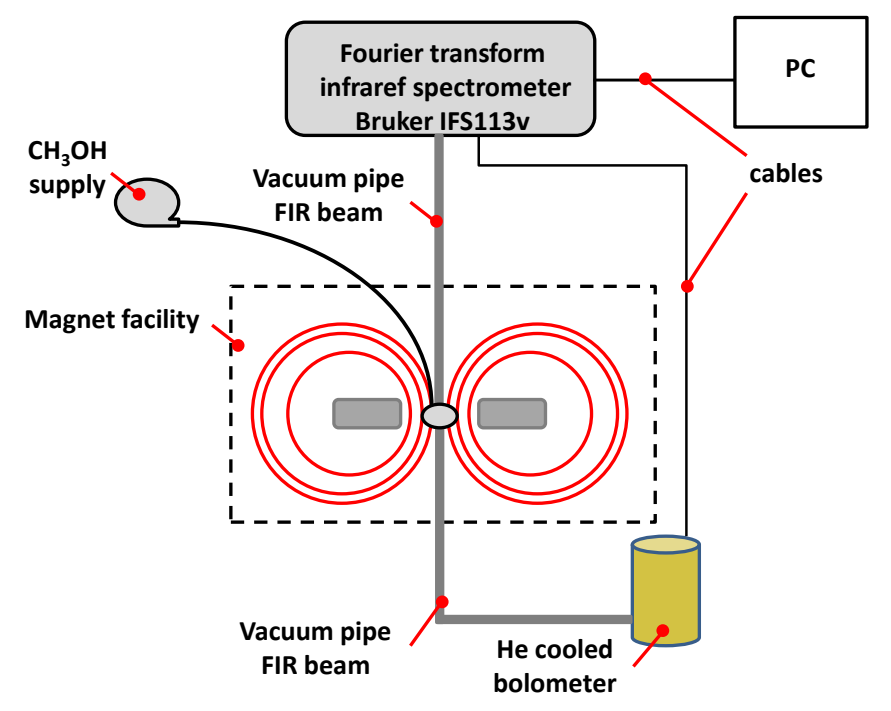

Figure 2: The scheme of the experimental apparatus built at the High Field Magnet Laboratory in Nijmegen (the Netherlands) to measure the Landé $g$-factors $\left(g_{\mathrm{L}}\right)$ of rotational $\mathrm{CH}_{3} \mathrm{OH} \mathrm{A}{ }^{+}$absorption lines in the frequency range between $0.6 \mathrm{THz}$ and $1.8 \mathrm{THz}$. The measured values could be used to deduce the $g_{\mathrm{L}}$ of the $5_{1}-6_{0} A^{+}$masing transition.

$1.8 \mathrm{THz}$ ). Because a preliminary study made in 1951 for the $g_{\mathrm{L}}$ of the E1-type $\mathrm{CH}_{3} \mathrm{OH}$ transitions showed that $g_{\mathrm{L}}^{\mathrm{E} 1}=0.078+1.88 /[J(J+1)][19]$, we suppose that the $g_{\mathrm{L}}$ will be of the order of 0.1 in the $A^{+}$transitions. In order to have a splitting of $\sim 0.05 \mathrm{THz}$, with an experimental resolution at most of $\sim 0.001 \mathrm{THz}$, magnetic fields up to $30 \mathrm{~T}$ were required. The scheme of the experimental apparatus that we used is shown in Fig. 2. A Fourier Transform Infrared Spectrometer generates a far-infrared beam that by traveling into a vacuum pipe passes through a sample holder, which is filled with $\mathrm{CH}_{3} \mathrm{OH}$ gas, that is located at the center of the magnet. Here, some of the infrared photons of the beam are absorbed, according to the rotational transitions of the molecule, by the $\mathrm{CH}_{3} \mathrm{OH}$ sample, whose pressure can be varied thanks to a vacuum pump and a $\mathrm{CH}_{3} \mathrm{OH}$ supply. The transmitted infrared beam is then detected by an helium-cooled bolometer and the detected signal is sent to a personal computer where the absorption spectrum is displayed. We made several measurements varying both the pressure of the $\mathrm{CH}_{3} \mathrm{OH}$ sample (from 10 to $60 \mathrm{mbar}$ ) and the magnetic field (from 0 to $30 \mathrm{~T}$ ). We observed neither the splitting nor the broadening of the absorption lines. The none detection of the Zeeman splitting could be due to two main aspects: (1) the splitting is smaller than the linewidth of the absorption lines, indicating that $g_{\mathrm{L}}$ for the $A^{+}$transitions is perhaps smaller than supposed; (2) the quadratic term of the Zeeman effect is not negligible already at small fields (few T). Because the measurements are very sensitive to the pressure of the $\mathrm{CH}_{3} \mathrm{OH}$ sample, to circumvent the aforementioned aspects we should design a new experiment where we use another source of radiation at lower frequencies or even a laboratory $\mathrm{CH}_{3} \mathrm{OH}$ maser placed at the center of the magnet, which should reach fields on the order of few Tesla.

However, there exists another way to estimate the $g_{\mathrm{L}}$ for the $6.7 \mathrm{GHz} \mathrm{CH} \mathrm{CH}_{3} \mathrm{OH}$ maser transition apart experimentally, that is by theoretically modeling the entire Zeeman effect of the complex $\mathrm{CH}_{3} \mathrm{OH}$ molecule. We contacted the Theoretical Chemistry group of the Institute for Molecules and Materials of the Radboud University who agreed to perform the computational calculations. At the moment of writing this proceeding, the Theoretical Chemistry group is finalizing the calcu- 
lations and the preliminary values of all the $\mathrm{CH}_{3} \mathrm{OH}$ molecule transitions, including all the masing transition, will soon be available.

\section{References}

[1] C.F. McKee \& E.C. Ostriker, Theory of Star Formation, ARA\&A, 45 (2007) 565 [arXiv:0707.3514].

[2] C.L.H. Hull, R.L. Plambeck, A.D Bolatto et al., Misalignment of Magnetic Fields and Outflows in Protostellar Cores, ApJ, 768 (2013) 159 [arXiv:1212.0540].

[3] N.L. Chapman, L.A. Davidson, P.F. Goldsmith et al. Alignment between Flattened Protostellar Infall Envelopes and Ambient Magnetic Fields, ApJ, 770 (2013) 151 [arXiv:1305.2922].

[4] C.F. McKee \& J. Tan, The Formation of Massive Stars from Turbulent Cores, ApJ 585 (2003) 850 [arXiv:astro-ph/0206037].

[5] T. Peters, R. Banerjee, R.S. Klessen et al., The Interplay of Magnetic Fields, Fragmentation, and Ionization Feedback in High-mass Star Formation, ApJ, 729 (2011) 72 [arXiv:1010.5905].

[6] D. Seifried, R.E. Pudritz, R. Banerjee et al., Magnetic fields during the early stages of massive star formation - II. A generalized outflow criterion, MNRAS, 422 (2012) 347 [arXiv:1109.4379].

[7] D. Seifried, R. Banerjee, R.E. Pudritz et al., Accretion and magnetic field morphology around Class 0 stage protostellar discs, 446 MNRAS, (2015) 2776 [arXiv:1408.2989].

[8] Q. Zhang, K. Qiu, J.M. Girart et al., Magnetic Fields and Massive Star Formation, ApJ, 792 (2014) 116 [arXiv:1407.3984]

[9] G. Surcis, W.H.T. Vlemmings, H.J. van Langevelde et al., EVN observations of $6.7 \mathrm{GHz}$ methanol maser polarization in massive star-forming regions II. First statistical results. A\&A, 556 (2013) 73 [arXiv:1306.6335].

[10] W.H.T. Vlemmings, R.M. Torres \& R. Dodson, Zeeman splitting of $6.7 \mathrm{GHz}$ methanol masers. On the uncertainty of magnetic field strength determinations, A\&A, $\mathbf{5 2 9}$ (2011) A95.

[11] G. Surcis, W.H.T. Vlemmings, H.J. van Langevelde et al., EVN observations of $6.7 \mathrm{GHz}$ methanol maser polarization in massive star-forming regions, A\&A, 541A (2012) 47 [arXiv:1203.4566].

[12] M.R. Pestalozzi, V. Minier \& R.S. Booth, A general catalogue of 6.7-GHz methanol masers. I. Data, $A \& A, 432$ (2005) 737 [arXiv:astro-ph/0411564].

[13] W.H.T. Vlemmings, G. Surcis, K.J.E. Torstensson et al., Magnetic field regulated infall on the disc around the massive protostar CepheusAHW2, MNRAS, 404 (2010) 134 [arXiv:1002.2214].

[14] G. Surcis, W.H.T. Vlemmings, R. Dodson et al., Methanol masers probing the ordered magnetic field of W75N, A\&A, $\mathbf{5 0 6}$ (2009) 757 [arXiv:0908.3585].

[15] G. Surcis, W.H.T. Vlemmings, R.M. Torres et al., The properties and polarization of the $\mathrm{H} 2 \mathrm{O}$ and CH3OH maser environment of NGC 7538-IRS 1 A\&A, 533A (2011) 47 [arXiv:1107.5313].

[16] G. Surcis, W.H.T. Vlemmings, H.J. van Langevelde et al., The magnetic field at milliarcsecond resolution around IRAS 20126+4104 A\&A, $\mathbf{5 6 3}$ (2014) A30 [arXiv:1401.7987].

[17] G. Surcis, W.H.T. Vlemmings, H.J. van Langevelde et al., EVN observations of $6.7 \mathrm{GHz}$ methanol maser polarization in massive star-forming regions III. The flux-limited sample., A\&A, submitted.

[18] R. Dodson \& C.D. Moriarty, Probing the magnetic fields of massive star-forming regions with methanol maser polarization, MNRAS, 421 (2012) 2395 [arXiv:1201.1687].

[19] C.K. Jen, Rotational Magnetic Moments in Polyatomic Molecules, Phys. Rev., 81 (1951) 197. 